Pacific Journal of Mathematics

Mooprusws Of GRour Extressio 


\title{
ISOMORPHISMS OF GROUP EXTENSIONS
}

\author{
KUNG-WEI YANG
}

To my parents

Let $0 \rightarrow G \rightarrow E \rightarrow \Pi \rightarrow 1$ and $0 \rightarrow G \rightarrow E^{\prime} \rightarrow \Pi \rightarrow 1$ be two crossed product extensions given by the crossed product groups $E=[G, \varphi, f, \Pi]$ and $E^{\prime}=\left[G, \varphi^{\prime}, f^{\prime}, \Pi\right]$ respectively. A homomorphism $\Gamma: E \rightarrow E^{\prime}$ is stabilizing if the diagram

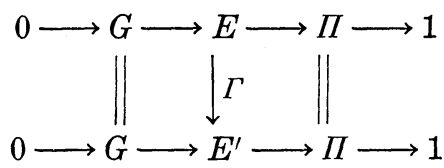

commutes. In this paper, a necessary and sufficient condition for the existence of a stabilizing homomorphism (hence isomorphism) between any two crossed product extensions is obtained.

The result is applied to obtain a necessary and sufficient condition for the existence of an automorphism $\Phi: E \rightarrow E$ making the diagram

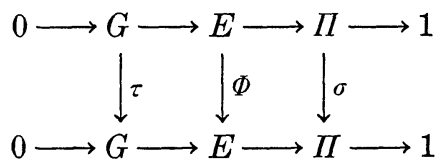

commutative, given $(\sigma, \tau) \in$ Aut $\Pi \times$ Aut $G$.

Notation. In general, we use the notation in [3]. Throughout the paper, $G$ and $\Pi$ denote two fixed groups. $G$ will be written in additive notation and $\Pi$ in multiplicative notation. Aut $G$, Out $G$, and $Z G$ are the automorphism group, the outer automorphism group, and the center of $G$, respectively. For any element $a \in G, \mu(a)$ denotes the inner automorphism $\mu(a)(g)=a+g-a$ given by conjugation with $a$. When $X$ is a group the natural image of an element $x \in X$ in a quotient group of $X$ is denoted $\bar{x}$. When $\varphi$ is a map, $\bar{\varphi}$ denotes the $\operatorname{map} \bar{\varphi}(x)=\overline{\varphi(x)}$.

Given groups $G, \Pi$, and functions $\varphi: \Pi \rightarrow$ Aut $G, f: \Pi \times \Pi \rightarrow G$ satisfying

$$
\begin{gathered}
\varphi(x) f(y, z)+f(x, y z)=f(x, y)+f(x y, z), \\
\varphi(x) \varphi(y)=\mu[f(x, y)] \varphi(x y),
\end{gathered}
$$

and the normalization conditions $\varphi(1)=1, f(x, 1)=0=f(1, y)$, the set $G \times \Pi$ under the sum defined by

$$
(g, x)+(h, y)=(g+\varphi(x) h+f(x, y), x y)
$$


is a group. The group so constructed is called a crossed product group, and is denoted $[G, \varphi, f, \Pi]$, or simply $E$. With the homomorphism $G \rightarrow E$ defined by $g \mapsto(g, 1)$, and $E \rightarrow I$ defined by $(g, x) \mapsto x$, we have an extension of $G$ by $\Pi$

$$
0 \longrightarrow G \longrightarrow E \longrightarrow \Pi \longrightarrow 1 \text {. }
$$

The extension is called a crossed product extension.

Results. Let $E=[G, \varphi, f, \Pi]$ and $E^{\prime}=\left[G, \varphi^{\prime}, f^{\prime}, \Pi\right]$ be two crossed product groups. Define a stabilizing homomorphism $\Gamma: E \rightarrow E^{\prime}$ as in the abstract. Notice that, by the "5 lemma" for groups, a stabilizing homomorphism is an isomorphism. Clearly, a homomorphism $\Gamma: E \rightarrow E^{\prime \prime}$ is stabilizing if and only if $\Gamma$ is of the form

$$
\Gamma(g, x)=(g+\gamma(x), x),
$$

and

$$
\varphi(x) g+f(x, y)+\gamma(x y)=\gamma(x)+\phi^{\prime}(x)[g+\gamma(y)]+f^{\prime}(x, y) .
$$

Because of the normalization conditions, $\gamma(1)=0$. Setting $y=1$ in (5), we obtain

$$
\varphi(x) g+\gamma(x)=\gamma(x)+\varphi^{\prime}(x) g .
$$

Setting $g=0$ in (5), we obtain

$$
f(x, y)+\gamma(x y)=\gamma(x)+\varphi^{\prime}(x) \gamma(y)+f^{\prime}(x, y) .
$$

Conversely, (5) can immediately be derived from (6) and (7). Summarizing, we have

Lemma. If $E=[G, \varphi, f, \Pi]$ and $E^{\prime}=\left[G, \varphi^{\prime}, f^{\prime}, \Pi\right]$ are two crossed product groups, then $\Gamma: E \rightarrow E^{\prime}$ is a stabilizing isomorphism if and only if $\Gamma$ is of the form (4), where the map $\gamma: \Pi \rightarrow G$ satisfies (6) and (7).

In particular, when $\varphi=\varphi^{\prime}$, and $f=f^{\prime}$, we see that by $(6), \gamma(x) \in$ $Z G$, and by (7), $\gamma \in Z^{1}(\Pi, Z G) . \quad Z^{1}(\Pi, Z G)$ is the group of normalized 1 -cocycles, and the $\Pi$-module structure on $Z G$ is given by $\varphi$.

CoRollary. If $E=[G, \varphi, f, \Pi]$ is a crossed product group, then $\Gamma: E \rightarrow E$ is a stabilizing automorphism if and only if $\Gamma$ is of the form (4), where $\gamma \in Z^{1}(\Pi, Z G)$.

We remark that both the lemma and the corollary are well-known. See for instance, [5, p. 127], [2, 17.1 Satz, p. 119], [4]. 
It is obvious from (6) that, as homomorphisms from $\Pi$ to Out $G$, (8)

$$
\bar{\varphi}=\bar{\varphi}^{\prime} \text {. }
$$

Trivially, (7) implies that

$$
k(x, y)=-f(x, y)+\gamma(x)+\phi^{\prime}(x) \gamma(y)+f^{\prime}(x, y)-\gamma(x y)
$$

is equal to 0 .

Conversely, given crossed product groups $E=[G, \varphi, f, \Pi]$ and $E=\left[G, \varphi^{\prime}, f^{\prime}, \Pi\right]$, we can ascertain the existence of a stabilizing isomorphism from $E$ to $E^{\prime}$ by the following procedure. First, we decide whether condition (8) is fulfilled. If not, the question is settled. If (8) is satisfied, then there is a function $\gamma: \Pi \rightarrow G$ such that (6) holds, and $Z G$ acquires a well-defined $\Pi$-module structure with operators $x c=\varphi(x) c\left(=\phi^{\prime}(x) c\right)$, for $c \in Z G$. We set $\gamma(1)=0$. It is now meaningful to speak of the group $Z^{2}(\Pi, Z G)$ (resp., $B^{2}(I, Z G)$ ) of the 2-dimensional normalized cocycles (resp., coboundaries) of $\Pi$ with values in $Z G$. Define $k(x, y)$ by (9). We claim that $k(x, y) \in Z^{2}(\Pi, Z G)$. Trivially, $k(x, 1)=0=k(1, y)$. To see $k(x, y) \in Z G$, we merely observe that conjugating $\varphi^{\prime}(x y) g$ with $\gamma(x y)$ and conjugating $\varphi^{\prime}(x y) g$ with $-f(x, y)+\gamma(x)+\varphi^{\prime}(x) \gamma(y)+f^{\prime}(x, y)$ give the same result $\varphi(x y) g$. $k(x, y)$, being the difference of these two elements, is therefore in $Z G$. To verify the identity

$$
x k(y, z)-k(x y, z)+k(x, y z)-k(x, y)=0,
$$

we observe that

$$
\begin{aligned}
k(x, y z) & -k(x y, z) \\
= & -f(x, y z)+\gamma(x)+\varphi^{\prime}(x) \gamma(y z)+f^{\prime}(x, y z)-f^{\prime}(x y, z) \\
& -\varphi^{\prime}(x y) \gamma(z)-\gamma(x y)+f(x y, z) \\
= & -f(x, y z)+\gamma(x)+\varphi^{\prime}(x)\left[\gamma(y z)-f^{\prime}(y, z)-\varphi^{\prime}(y) \gamma(z)\right] \\
& +f^{\prime}(x, y)-\gamma(x y)+f(x y, z) \\
= & -f(x, y z)+\gamma(x)-\varphi^{\prime}(x) k(y, z)-\varphi^{\prime}(x) f(y, z)-\gamma(x) \\
& +f(x, y)+k(x, y)+f(x y, z) \\
= & k(x, y)-x k(y, z) .
\end{aligned}
$$

We made use of the identities (1), (2), (9), (6), and (1), in that order.

Finally, if $k(x, y) \in B^{2}(\Pi, Z G)$, then $k(x, y)=x \beta(y)-\beta(x y)+\beta(x)$. The function $\gamma^{\prime}: \Pi \rightarrow G$ defined by $\gamma^{\prime}(x)=\gamma(x)-\beta(x)$ satisfies (6) and (7). Therefore, the map $\Gamma$ defined by (4), using $\gamma^{\prime}$ instead of $\gamma$, is a stabilizing isomorphism. If $k(x, y) \notin B^{2}(\Pi, Z G)$, then there could not exist a stabilizing isomorphism $\Gamma: E \rightarrow E^{\prime}$. For, if there were to exist such an isomorphism, the discussion leading up to the above lemma would show that $\Gamma(g, x)=\left(g+\gamma^{\prime}(x), x\right)$, with $\gamma^{\prime}$ satisfying (6) and (7). 
Since $\gamma$ and $\gamma^{\prime}$ both satisfy (6), $\beta(x)=\gamma(x)-\gamma^{\prime}(x) \in Z G$. By (7) we have $k(x, y)=x \beta(y)-\beta(x y)+\beta(x)$ showing $k(x, y) \in B^{2}(\Pi, Z G)$. This discussion also shows that $\overline{k(x, y)}$ in $H^{2}(\Pi, Z G)$ is independent of the choice of $\gamma$. These results may now be summarized as follows.

Theorem 1. Let $E=[G, \varphi, f, \Pi]$ and $E^{\prime}=\left[G^{\prime}, \varphi^{\prime}, f^{\prime}, \Pi\right]$ be two crossed product groups. Then there exists a stabilizing isomorphism $\Gamma: E \rightarrow E^{\prime}$, if and only if

(A) $\bar{\varphi}=\bar{\varphi}^{\prime}$, and

(B) $\bar{k}=0$ in $H^{2}(I I, Z G)$, where $k(x, y)$ is defined as above.

We note that Theorem 1 is well-known (and is easily seen to be true) in the case where $\varphi=\varphi^{\prime}$ [3, Theorem 8.8, p. 128, Lemma 8.2].

An application. Let $0 \rightarrow G \rightarrow E \rightarrow \Pi \rightarrow 1$ be a group extension. Call an automorphism of $E$ taking $G$ onto $G$ an automorphism over $G$. Clearly, any automorphism of $E$ over $G$ induces automorphisms $\tau$ on $G$ and $\sigma$ on $\Pi$. It is easy to see that, in general, not every pair $(\sigma, \tau) \in$ Aut $\Pi \times$ Aut $G$ can be so induced by an automorphism of $E$ over $G$. In [4], Charles Wells defined an exact sequence which gives a necessary and sufficient condition for a pair $(\sigma, \tau) \in$ Aut $\Pi \times$ Aut $G$ to be inducible by an automorphism of $E$ over $G$. We now apply Theorem 1 to prove a similar result. We hope our method will also help clarify the nature of the map $C \rightarrow H_{\alpha}^{2}(\Pi, Z G)$ as defined in [4].

Let $0 \rightarrow G \rightarrow E \rightarrow \Pi \rightarrow 1$ be a group extension. We may (and do) assume that $E$ is of the form $E=[G, \varphi, f, \Pi]$ with homomorphisms $G \rightarrow E, E \rightarrow I I$ of the form as defined in the definition of a crossed product extension at the beginning of this paper. We say that a pair $(\sigma, \tau) \in$ Aut $I \times$ Aut $G$ is inducible if there exists an automorphism $\Phi: E \rightarrow E$ such that the diagram

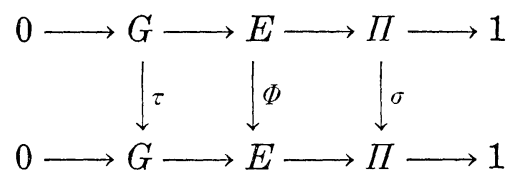

is commutative.

For $(\sigma, \tau) \in$ Aut $\Pi \times$ Aut $G$, let $\varphi_{\sigma}(x)=\varphi(\sigma x), f_{\sigma}(x, y)=f(\sigma x, \sigma y)$; $\varphi_{\tau}(x)=\tau \varphi(x) \tau^{-1}, f_{\tau}(x, y)=\tau f(x, y)$. If $\bar{\varphi}_{\sigma}=\bar{\varphi}_{\bar{\tau}}$, there exists a map $\gamma: \Pi \rightarrow G$ such that $\varphi_{\tau}(x)+\gamma(x)=\gamma(x)+\varphi_{\sigma}(x)$. Choose $\gamma$ so that $\gamma(1)=0$. In this case, define

$$
k_{\sigma,-}(x, y)=-f_{\tau}(x, y)+\gamma(x)+\varphi_{\sigma}(x) \gamma(y)+f_{\sigma}(x, y)-\gamma(x y) .
$$

THEOREM 2. The pair $(\sigma, \tau) \in$ Aut $\Pi \times$ Aut $G$ is inducible if and 
only if

(A) $\bar{\varphi}_{o}=\bar{\varphi}_{\tau}$, and

(B) $\bar{k}_{o, \tau}=0$ in $H^{2}(\Pi, Z G)$,

where $k_{\sigma, \tau}(x, y)$ is defined as in (11), and the $\Pi$-module structure on $Z G$ is induced by the homomorphism $\bar{\varphi}_{\sigma}=\bar{\varphi}_{\tau}$.

Proof. Let $0 \rightarrow G \rightarrow E \rightarrow \Pi \rightarrow 1$ be a group extension. Set $E=$ $[G, \varphi, f, \Pi]$. Let $\varphi_{o}, f_{o}, \varphi_{\tau}, f_{\tau}, k_{\sigma, \tau}$ be as defined in the paragraph preceding Theorem 2. Let $E_{\tau}=\left[G, \varphi_{\tau}, f_{\tau}, \Pi\right], E_{\sigma}=\left[G, \varphi_{o}, f_{\sigma}, \Pi\right]$. Define $T: E \rightarrow E_{\tau}$ by $T(g, x)=(\tau g, x)$, and $\Sigma: E_{\sigma} \rightarrow E$ by $\Sigma(g, x)=(g, \sigma x)$. It is a straightforward matter to check that $T$ and $\Sigma$ are both group homomorphisms and that the following two diagrams

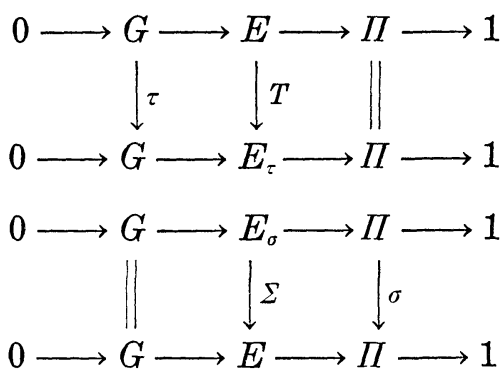

commute.

If there is a stabilizing isomorphism $\Gamma: E_{\tau} \rightarrow E_{\sigma^{\prime}}$ then $(\sigma, \tau)$ is clearly inducible.

Conversely, if $(\sigma, \tau)$ is inducible, then there exists an automorphism $\Phi: E \rightarrow E$ such that the diagram (10) is commutative. Such an automorphism is necessarily of the form $\Phi(g, x)=(\tau g+\gamma(x), \sigma x)$, where $\gamma$ satisfies (6) and (7) with $\varphi$ replaced by $\varphi_{\tau}$ and $\varphi^{\prime}$ replaced by $\varphi_{\sigma}$. Therefore, $\Gamma=\Sigma^{-1} \Phi T^{-1}$ is a stabilizing isomorphism from $E_{\tau}$ to $E_{\sigma}$. By Theorem 1, $(\sigma, \tau)$ is inducible if and only if (A) and (B) are satisfied.

Condition (A) of Theorem 2 can be stated more explicitly as follows: For any $x \in \Pi, \bar{\tau} \overline{\varphi(x)} \bar{\tau}^{-1}=\overline{\varphi(\sigma x)}$.

As direct consequences of Theorem 2, we have

Corollary 1. If Out $G=1$ and $H^{2}(\Pi, Z G)=0$, then every pair $(\sigma, \tau) \in$ Aut $\Pi \times$ Aut $G$ is inducible.

Corollary 2. If Out $G=1$ and $Z G=0$, then for any group $E$ such that $G \triangleleft E$ and for each $\tau \in$ Aut $G$, there exists $\Phi \in$ Aut $E$ such that restriction of $\Phi$ to $G$ is equal to $\tau$.

The second corollary also follows directly from [1, Theorem 1]. 


\section{REFERENCES}

1. R. Baer, Absolute retracts in group theory, Bull. Amer. Math. Soc., 52 (1946), 501-6.

2. B. Huppert, Endliche Gruppen I, Die Grundlehren der math. Wissenschaften, Band 134, Springer-Verlag, Berlin and New York, 1967.

3. S. MacLane, Homology, Die Grundlehren der math. Wissenschaften, Band 114, Academic Press, New York, Springer Verlag, Berlin, Grottingen, Heidelberg, 1963.

4. C. Wells, Automorphisms of group extensions, Trans. Amer. Math. Soc., 155 (1970), 189-194.

5. H. J. Zassenhaus, The Theory of Groups, 2nd ed., Chelsea Publishing Company, 1958.

Received September 28, 1972.

Western Michigan UNIVERSity 


\section{PACIFIC JOURNAL OF MATHEMATICS}

\section{EDITORS}

RICHARD ARENS (Managing Editor) University of California

Los Angeles, California 90024

R. A. BeaUmont

University of Washington Seattle, Washington 98105
J. DUGUNDJI*

Department of Mathematics University of Southern California Los Angeles, California 90007

D. Gilbarg and J. Milgram Stanford University

Stanford, California 94305

\section{ASSOCIATE EDITORS}

E. F. BECKENBACH

B. H. NeUmanN

F. WOLF

K. YoSHIDA

\section{SUPPORTING INSTITUTIONS}

\section{UNIVERSITY OF BRITISH COLUMBIA CALIFORNIA INSTITUTE OF TECHNOLOGY UNIVERSITY OF CA.LIFORNIA MONTANA STATE UNIVERSITY UNIVERSITY OF NEVADA NEW MEXICO STATE UNIVERSITY OREGON STATE UNIVERSITY UNIVERSITY OF OREGON OSAKA UNIVERSITY}

UNIVERSITY OF SOUTHERN CALIFORNIA STANFORD UNIVERSITY UNIVERSITY OF TOKYO UNIVERSITY OF UTAH WASHINGTON STATE UNIVERSITY UNIVERSITY OF WASHINGTON AMERICAN MATHEMATICAL SOCIETY NAVAL WEAPONS CENTER

* C. R. DePrima California Institute of Technology, Pasadena, CA 91109, will replace J. Dugundji until August 1974. 


\section{Pacific Journal of Mathematics}

\section{Vol. 50, No. $1 \quad$ September, 1974}

Gail Atneosen, Sierpinski curves in finite 2-complexes.............. 1

Bruce Alan Barnes, Representations of $B^{*}$-algebras on Banach spaces .... 7

George Benke, On the hypergroup structure of central $\Lambda(p)$ sets ....... 19

Carlos R. Borges, Absolute extensor spaces: a correction and an

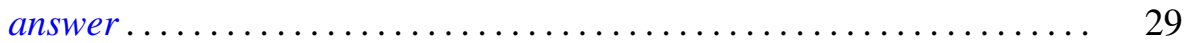

Tim G. Brook, Local limits and tripleability .................. 31

Philip Throop Church and James Timourian, Real analytic open maps .... 37

Timothy V. Fossum, The center of a simple algebra ............... 43

Richard Freiman, Homeomorphisms of long circles without periodic

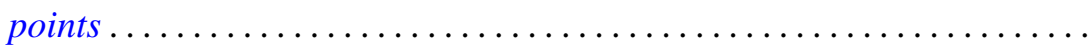

B. E. Fullbright, Intersectional properties of certain families of compact

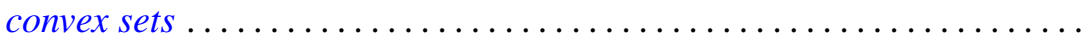

Harvey Charles Greenwald, Lipschitz spaces on the surface of the unit

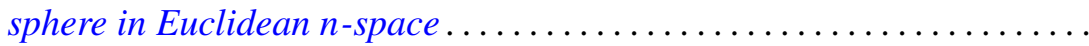

Herbert Paul Halpern, Open projections and Borel structures for

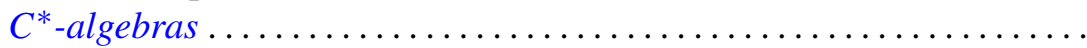

Frederic Timothy Howard, The numer of multinomial coefficients divisible

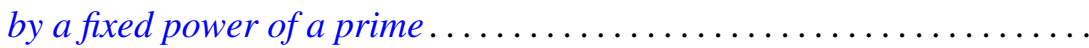

Lawrence Stanislaus Husch, Jr. and Ping-Fun Lam, Homeomorphisms of manifolds with zero-dimensional sets of nonwandering points........ 109

Joseph Edmund Kist, Two characterizations of commutative Baer rings ...

Lynn McLinden, An extension of Fenchel's duality theorem to saddle functions and dual minimax problems ................

Leo Sario and Cecilia Wang, Counterexamples in the biharmonic classification of Riemannian 2-manifolds...

Saharon Shelah, The Hanf number of omitting complete types ...

Richard Staum, The algebra of bounded continuous functions into a

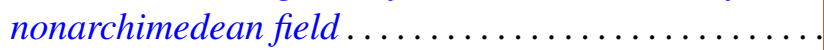

James DeWitt Stein, Some aspects of automatic continuity ..

Tommy Kay Teague, On the Engel margin

John Griggs Thompson, Nonsolvable finite groups all of whose local subgroups are solvable, $V \ldots \ldots \ldots \ldots \ldots \ldots \ldots \ldots$

Kung-Wei Yang, Isomorphisms of group extensions 